\author{
G. Perseghin - A. Burska - G. Lattuada - G. Alberti • \\ F. Costantino - F. Ragogna $\cdot$ S. Oggionni $\cdot$ A. Scollo $\cdot$ \\ I. Terruzzi $\cdot$ L. Luzi
}

\title{
Increased serum resistin in elite endurance athletes with high insulin sensitivity
}

Received: 10 February 2006 / Accepted: 12 March 2006 / Published online: 10 May 2006

(C) Springer-Verlag 2006

\begin{abstract}
Aims/hypothesis: Resistin is an adipokine associated with obesity and type 2 diabetes in animal models, but in humans its role remains uncertain. This study was undertaken to test whether serum resistin is related to insulin resistance and markers of low-grade inflammation in elite athletes taken as a model of extreme insulin sensitivity. Subjects materials and methods: In 23 elite athletes (sprinters, middle-distance and marathon runners) and in 72 sedentary men including lean and obese individuals with NGT, and obese individuals with IGT or new-onset type 2 diabetes, we assessed insulin sensitivity using a whole-body insulin-sensitivity index (WBISI) derived from a 3-h OGTT; energy homeostasis was also assessed by means of indirect calorimetry, along with circulating adipokines and low-grade pro-inflammatory cyto-chemokines. Results: Professional athletes had increased WBISIs $(p<0.001)$ and lipid oxidation $(p<0.03)$; they also showed higher serum resistin concentrations $(p<0.001)$, although the pro-inflammatory chemokines were not increased in comparison with the other study groups. Resistin was independently associated only with fasting plasma NEFA. Increased resistin was detected in the middle-distance and marathon runners, but not in the sprinters when compared with the lean, young,
\end{abstract}

G. Perseghin $(\bowtie) \cdot$ A. Burska $\cdot$ G. Lattuada $\cdot$ F. Costantino F. Ragogna - S. Oggionni - A. Scollo · I. Terruzzi - L. Luzi Internal Medicine, Section of Nutrition/Metabolism and Unit of Clinical Spectroscopy, San Raffaele Scientific Institute,

via Olgettina 60,

20132 Milan, Italy

e-mail: perseghin.gianluca@hsr.it

Tel.: +39-02-26432614

Fax: +39-02-26432771

G. Alberti · L. Luzi

Institute of Physical Exercise, Health and Sport Activities,

Faculty of Exercise Sciences, University of Milan,

Milan, Italy

G. Perseghin · G. Alberti · L. Luzi

Center for Physical Exercise for Health and Wellness,

University of Milan,

Milan, Italy sedentary individuals. Conclusions/interpretation: Serum resistin concentration is increased in elite athletes, providing evidence against the notion that resistin levels reflect insulin resistance in humans, as seen in animal studies. Increased resistin was observed in aerobicendurance, but not sustained-power athletes and this feature appeared to be independently associated with parameters of fatty acid metabolism.

Keywords Adipokines - Lipid oxidation - NEFA · OGTT · Physical exercise

Abbreviations CCL2: chemoattractant CC chemokine ligand 2 - HOMA: homeostasis model assessment - MCP: macrophage chemotactic protein - MIP: macrophage inflammatory protein - REE: resting energy expenditure RQ: respiratory quotient - WBISI: whole-body insulinsensitivity index

\section{Introduction}

Administration of resistin to mice impairs glucose tolerance [1] and alters insulin-mediated hepatic gluconeogenesis [2], while neutralisation with anti-resistin antibody improves insulin sensitivity [1]. In addition, adenovirusmediated chronic 'hyper-resistinaemia' induced in vivo insulin resistance in normal rats [3] and hepatic insulin resistance in high-fat-fed mice [4]. Based on this research in animal models, it was hypothesised that resistin might represent the link between obesity and insulin resistance. In spite of many efforts, little is known about the function of resistin in humans [5]. The identity of resistin amino acid sequences in mouse and human proteins is only $59 \%$ [6] and because of this incomplete homology resistin may have different physiological roles in different species [7]. In fact, studies in humans reported conflicting results, indicating decreased or increased resistin levels in obesity, opposite responses to insulin-sensitisers and contradictory correlations between resistin and markers of insulin resistance [8-15]. It has also been suggested that resistin plays a role 
in inflammatory conditions, because it is expressed in human macrophages [16] and because recent data have supported the existence of an independent association of resistin with the inflammatory factors IL-6 and C-reactive protein [17]. The aim of the present study was to examine whether resistin is a marker of insulin resistance or lowgrade inflammation in a human model of extreme insulin sensitivity, i.e. elite athletes, when compared not only with insulin-resistant individuals but also with normal healthy individuals.

\section{Subjects, materials and methods}

Subjects

Twenty-three elite track runners (sprinters: $n=6$; middledistance: $n=7$; endurance athletes: $n=10$ ) who participate in national and international competitions were recruited within the Federazione Italiana Atletica Leggera-Lombardia Section. They were compared with 13 sedentary lean young men. The study also included middle-aged overweight individuals with NGT $(n=34)$ and overweight individuals with IGT $(n=12)$ or with new-onset type 2 diabetes $(n=13)$ as classic control groups characterised by varying degrees of insulin resistance. Glucose-tolerance status was established on the basis of a 75-g OGTT performed in accordance with the criteria suggested by the American Diabetes Association. The non-athletic indi- viduals were recruited in the outpatient services of the Center of Nutrition/Metabolism of the San Raffaele Scientific Institute. Body weight was stable for at least 6 months. The anthropometric characteristics of the subjects are summarised in Table 1. All subjects were in good health as assessed by medical history, physical examination and haematological and urine analysis. Informed consent was obtained from all subjects after explanation of the purpose, nature and potential risks of the study. The protocol was approved by the Ethical Committee of the San Raffaele Scientific Institute.

\section{Experimental protocol}

Subjects were instructed to consume an isocaloric diet and to abstain from exercise activity for 3 days before the studies.

OGTT Subjects were admitted to the Metabolic Room of the Section of Nutrition/Metabolism of the San Raffaele Scientific Institute at $07.30 \mathrm{~h}$ after a 10 -h overnight fast. The OGTT was performed at $08.00-08.30 \mathrm{~h}$ following the American Diabetes Association recommendations [18]. A Teflon catheter was inserted into an antecubital vein for blood sampling; blood was obtained for the assessment of glucose, insulin and NEFA concentrations in the basal period and after the oral glucose load (75 g) at 30-min intervals for $180 \mathrm{~min}$. Samples were also collected for the

Table 1 Anthropometric and laboratory features and insulin sensitivity of study groups

\begin{tabular}{|c|c|c|c|c|}
\hline & Athletes & Lean NGT & Overweight NGT & Overweight IGT/T2DM \\
\hline$n$ & 23 & 13 & 34 & 25 \\
\hline \multicolumn{5}{|l|}{ Anthropometry } \\
\hline Age (years) & $24 \pm 5^{\mathrm{a}}$ & $26 \pm 4^{\mathrm{a}}$ & $49 \pm 9$ & $49 \pm 9$ \\
\hline Body weight (kg) & $69 \pm 7^{\mathrm{a}}$ & $69 \pm 7^{\mathrm{a}}$ & $84 \pm 17$ & $87 \pm 18$ \\
\hline Height $(\mathrm{cm})$ & $179 \pm 6$ & $176 \pm 6$ & $173 \pm 6$ & $174 \pm 6$ \\
\hline BMI $\left(\mathrm{kg} / \mathrm{m}^{2}\right)$ & $21.5 \pm 1.2^{\mathrm{a}}$ & $22.5 \pm 2.4^{\mathrm{a}}$ & $28.0 \pm 6.0$ & $28.6 \pm 4.8$ \\
\hline \multicolumn{5}{|l|}{ Laboratory } \\
\hline Fasting NEFA (mmol/l) & $0.65 \pm 0.24$ & $0.50 \pm 0.22$ & $0.55 \pm 0.18$ & $0.59 \pm 0.19$ \\
\hline Total cholesterol (mmol/l) & $3.7 \pm 0.7^{\mathrm{a}}$ & $4.2 \pm 1.0^{\mathrm{a}}$ & $5.6 \pm 1.0$ & $5.9 \pm 1.3$ \\
\hline HDL-cholesterol (mmol/l) & $1.5 \pm 0.3^{\mathrm{a}}$ & $1.4 \pm 0.4^{\mathrm{b}}$ & $1.1 \pm 0.3$ & $1.1 \pm 0.2$ \\
\hline LDL-cholesterol (mmol/l) & $1.9 \pm 0.6^{\mathrm{a}}$ & $2.4 \pm 0.9^{\mathrm{a}}$ & $3.6 \pm 0.8$ & $3.9 \pm 1.2$ \\
\hline Triglycerides (mmol/l) & $0.7 \pm 0.2^{\mathrm{a}}$ & $1.0 \pm 0.5^{\mathrm{b}}$ & $2.5 \pm 0.9$ & $2.0 \pm 1.0$ \\
\hline Total homocysteine $(\mu \mathrm{mol} / \mathrm{l})$ & $12.0 \pm 4.7$ & $8.9 \pm 2.0$ & $11.0 \pm 4.0$ & $11.6 \pm 2.9$ \\
\hline \multicolumn{5}{|l|}{ OGTT } \\
\hline Fasting glucose (mmol/1) & $4.7 \pm 0.3^{\mathrm{a}}$ & $4.9 \pm 0.4^{\mathrm{c}}$ & $5.5 \pm 0.4^{\mathrm{c}}$ & $6.6 \pm 1.4$ \\
\hline Mean glucose $\mathrm{e}_{30-180}(\mathrm{mmol} / \mathrm{l})$ & $5.4 \pm 0.7^{\mathrm{b}}$ & $5.5 \pm 0.6^{\mathrm{c}}$ & $6.7 \pm 0.9^{\mathrm{c}}$ & $10.5 \pm 3.1$ \\
\hline Fasting insulin $(\mathrm{pmol} / \mathrm{l})$ & $46 \pm 27^{\mathrm{a}}$ & $72 \pm 36$ & $86 \pm 60$ & $93 \pm 54$ \\
\hline Mean insulin ${ }_{30-180}(\mathrm{pmol} / \mathrm{l})$ & $141 \pm 44^{\mathrm{a}}$ & $252 \pm 80$ & $378 \pm 203$ & $369 \pm 209$ \\
\hline WBISI & $9.77 \pm 3.87^{\mathrm{a}, \mathrm{d}}$ & $5.49 \pm 2.46^{\mathrm{c}}$ & $4.21 \pm 2.22$ & $3.02 \pm 1.64$ \\
\hline
\end{tabular}

Values are means \pm SD

By one-way ANOVA and post hoc Bonferroni:

${ }_{p} p<0.001$ vs overweight NGT and overweight IGT/type 2 diabetes mellitus (T2DM)

${ }^{\mathrm{b}} p<0.05$ vs overweight NGT and overweight IGT/T2DM

${ }^{c} p<0.01$ vs overweight IGT/T2DM

${ }_{p}<0.01$ vs lean NGT 
assessment of fasting plasma or serum concentration of total cholesterol, HDL-cholesterol, triglycerides, leptin, adiponectin, resistin, macrophage inflammatory protein (MIP)-1 $\alpha$, MIP-1 $\beta$, macrophage chemotactic protein (MCP)-1, IL-6 and total homocysteine.

Indirect calorimetry Indirect calorimetry was performed continuously, while lying quietly, for $30 \mathrm{~min}$ during the basal equilibration period with a ventilated hood system (Sensor Medics 2900; Metabolic Measurement Cart) to measure $\mathrm{VO}_{2}$ and $\mathrm{VCO}_{2}$ to calculate glucose and lipid oxidation. The mean $\mathrm{CV}$ values within the session for both $\mathrm{O}_{2}$ and $\mathrm{CO}_{2}$ measurements were below $2 \%$.

Serum resistin in middle-age, non-professional endurance-trained individuals

To test whether the serum resistin pattern characterising elite professional athletes was comparable with that of nonprofessional but physically active individuals, its fasting serum concentration was measured in a group of middleage men $\left(n=9\right.$, age $47 \pm 11$ years, BMI $\left.22.7 \pm 1.3 \mathrm{~kg} / \mathrm{m}^{2}\right)$ involved in amateur endurance-training programmes and was compared with that of sedentary individuals matched for anthropometric features $(n=9$, age $42 \pm 8$ years, BMI $22.5 \pm 0.9 \mathrm{~kg} / \mathrm{m}^{2}$ ). These study subjects were not originally part of the present study, but were part of another study in which cardiac function and metabolism were assessed using magnetic resonance techniques in middle-age men undergoing regular aerobic exercise training in comparison with matched individuals with a sedentary life-style (data not shown). Resistin was measured retrospectively in these individuals to look for a potential confirmation of the data observed in the elite athletes.

\section{Analytical determinations}

Glucose concentration was measured with a standard glucose oxidase method on a glucose analyser (Beckman Coulter, Fullerton, CA, USA). Blood samples for NEFA assessment were collected in pre-chilled tubes containing $0.1 \%$ EDTA. Tubes were immediately placed in ice and plasma was immediately processed by centrifugation at $4^{\circ} \mathrm{C}$. Plasma was then frozen and stored at $-70^{\circ} \mathrm{C}$ and NEFA determinations were performed within $0-5$ working days as suggested by Zambon and coworkers to obtain plasma NEFA determinations avoiding lipoprotein lipase reactions in blood [19]. Serum triglycerides, total cholesterol and HDL-cholesterol were measured as previously described [20]. LDL-cholesterol was calculated using the Friedwald formula. The total plasma homocysteine level was determined using an HPLC method based on the derivatisation with SBD-F (ammonium-7-fluorobenzo-2-oxa-1,3-diazole4-sulphonate) fluorescence as previously described [21]. Serum resistin was measured with an ELISA kit (BioVendor Laboratory Medicine, Brno, Czech Republic). The sensitivity of the assay was $0.2 \mathrm{ng} / \mathrm{ml}$ of sample. The intra-assay CV was $<3.5 \%$ and the interassay $<7 \%$. Plasma levels of insulin (sensitivity $2 \mu \mathrm{U} / \mathrm{ml}$; intra- and interassay $\mathrm{CV}$ values $<3.1$ and $6 \%$, respectively) and leptin (sensitivity $0.5 \mathrm{ng} / \mathrm{ml}$; intraand interassay $\mathrm{CV}$ values $<5$ and $9 \%$, respectively) were measured with an RIA (Linco Research, St Charles, MO, USA) following the manufacturer's assay protocols. Serum adiponectin was measured by an ELISA kit (B-Bridge International, Sunnyvale, CA, USA) with a sensitivity of $25 \mathrm{pg} / \mathrm{ml}$. The intra-assay CV was $<3.7 \%$ and the interassay $<6 \%$. Serum markers of inflammation, MCP-1, IL-6, MIP$1 \alpha$ and MIP- $1 \beta$ (sensitivity $1.5,0.2,6.25$ and $0.8 \mathrm{pg} / \mathrm{ml}$, respectively), were measured with a Human Chemokine/ Cytokine Array-SearchLight assay (Pierce Biotechnology, Rockford, IL, USA).

\section{Calculations}

The whole-body insulin-sensitivity index (WBISI) was measured using the modified formula of Matsuda and DeFronzo [22] using the results obtained by the assessments in all seven blood samples collected during the 3-h OGTT:

$$
\begin{aligned}
& \mathrm{ISI}_{(\mathrm{OGTT})} \\
& =\frac{10,000}{\sqrt{\left(G_{0} \times I_{0}\right) \times\left(\text { mean } G_{30-180 \mathrm{~min}}\right) \times\left(\text { mean } I_{30-180 \mathrm{~min}}\right)}}
\end{aligned}
$$

where $I_{0}(\mu \mathrm{U} / \mathrm{ml})$ was the fasting insulin concentration, $G_{0}$ $(\mathrm{mg} / \mathrm{dl})$ was the fasting glucose concentration and $G_{30}$

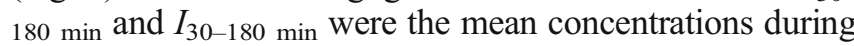
the 3-h OGTT of glucose and insulin, respectively. The groups of amateur endurance-trained middle-age men and the control group of sedentary individuals did not undergo the OGTT. In these groups, insulin sensitivity was estimated by means of an updated computer model of the homeostasis model assessment (HOMA)-2 indices [23] available from www.OCDEM.ox.ac.uk. Resting energy expenditure (REE) was calculated by Weir's standard equation [24] from the $\mathrm{O}_{2}$ consumption rate and the $\mathrm{CO}_{2}$ production rates measured by means of indirect calorimetry (excluding the first $10 \mathrm{~min}$ of data acquisition), and from the urinary nitrogen excretion. Predicted REE was calculated using the Harris-Benedict equations [25]. Glucose, lipid and protein oxidation were estimated as previously described [26].

\section{Statistical analysis}

Data in text, tables and figures are means \pm SD. Analysis were performed using SPSS software (version 10.0; SPSS, Chicago, IL, USA). Comparisons among all groups were performed using one-way ANOVA with a Bonferroni post hoc test, and a $p$ value less than 0.05 was considered to be statistically significant as shown in Tables 1,2 and 3 . Comparison between the middle-age, amateur endurancetrained men and matched sedentary controls was performed using a two-tailed independent-samples $t$-test as 
shown in Table 4 and a $p$ value less than 0.05 was considered to be statistically significant. When parameters showed a skewed distribution (Kolmogorov-Smirnov test of normality), they were log-transformed before the analysis (resistin, BMI, leptin, glucose, MCP-1/ chemoattractant CC chemokine ligand 2[CCL2], insulin, triglycerides, adiponectin and IL-6). Two-tailed Pearson's correlation was performed to establish partial correlation coefficients between resistin and other parameters. The anthropometric and some of the metabolic features of the sedentary individuals were homogeneous, while some of those of the elite athletes were rather peculiar, as already reflected in the comparison between groups. Based on this observation, and to assess whether the correlation analysis may add information beyond what was predictable from differences between groups, a partial correlation controlling for the group variable was repeated to establish groupindependent associations. A prior power calculation analysis using an optimal allocation strategy indicated that 20 athletes and 15 sedentary controls were required to provide a power of $90 \%$ to detect a $20 \%$ difference in serum resistin and 15 athletes and 15 sedentary controls were required to provide a power of $90 \%$ to detect a $30 \%$ difference in WBISI between groups.

\section{Results}

Anthropometric and laboratory characteristics of study subjects Anthropometric and laboratory features of the elite athletes are summarised in Table 1. The young lean men with NGT served as a reference group controlling for insulin sensitivity in sedentary, otherwise healthy normal individuals, and were matched for age and body weight with the group of elite athletes. The overweight individuals with NGT and IGT (including both IGT and type 2 diabetic patients) served as control groups with varying degrees of insulin resistance. It is obvious that despite the fact that the athletes and the lean sedentary NGT men were not different for age and BMI, body composition of these two groups of subjects must be different. In contrast, overweight men with NGT and IGT/type 2 diabetes mellitus were tightly matched for the anthropometric features. While fasting plasma NEFAs were not different among groups, the groups of overweight men (NGT and IGT/type 2 diabetes mellitus) had significantly higher total cholesterol, LDL-cholesterol and triglycerides and lower HDL-cholesterol than the athletes and the lean sedentary controls (Table 1).

OGTT Plasma glucose was not different between the athletes and the lean young controls in the fasting state and during the OGTT (as reflected by the mean glucose concentration) (Table 1). All the groups of overweight men had higher fasting plasma glucose in comparison with athletes and the lean sedentary controls and the mean glucose concentration was graded with the glucosetolerance status (Table 1). Fasting plasma insulin concentration was not different between the athletes and the lean sedentary controls but was lower in the athletes in comparison with overweight NGT and IGT/type 2 diabetic men (Table 1). The mean insulin concentration during the OGTT was significantly lower in athletes in comparison with NGT and IGT/type 2 diabetic patients (Table 1). Based on the surrogate index of insulin sensitivity (WBISI) the athletes were more insulin-sensitive with respect to both the overweight men (NGT and IGT/type 2 diabetes mellitus; $p<0.001$ ) and the lean sedentary controls (Table $1 ; p<0.01$ ).

Adipokines Plasma leptin was significantly lower in the athletes in comparison with all the other study groups (Table 2). In spite of comparable BMIs, the lower leptin levels in the athletes in comparison with the lean sedentary controls would suggest a reduced body fat in the former. Serum adiponectin was not different among groups (Table 2). Serum resistin concentrations were significantly higher in the athletes in comparison with both the lean sedentary controls $(p<0.001$; Fig. 1a) and the overweight groups (NGT, IGT/type 2 diabetes mellitus; $p<0.0001$; Fig. 1a).

Table 2 Adipocyte hormones and pro-inflammatory chemokines in study groups

\begin{tabular}{lcccc}
\hline & Athletes & Lean NGT & Overweight NGT & Overweight IGT/T2DM \\
\hline$n$ & 23 & 13 & 34 & 25 \\
Leptin $(\mathrm{ng} / \mathrm{ml})$ & $1.3 \pm 0.5^{\mathrm{a}, \mathrm{d}}$ & $3.7 \pm 2.3$ & $7.6 \pm 6.8$ & $7.2 \pm 4.8$ \\
Adiponectin $(\mu \mathrm{g} / \mathrm{ml})$ & $8.0 \pm 2.9$ & $6.9 \pm 3.2$ & $6.4 \pm 1.7$ & $6.7 \pm 3.8$ \\
Resistin $(\mathrm{ng} / \mathrm{ml})$ & $4.5 \pm 1.6^{\mathrm{a}, \mathrm{d}}$ & $3.1 \pm 0.7$ & $3.0 \pm 0.6$ & $2.6 \pm 0.7$ \\
MCP-1/CCL2 $(\mathrm{pg} / \mathrm{ml})$ & $221 \pm 95^{\mathrm{c}}$ & $262 \pm 72$ & $281 \pm 102$ & $334 \pm 84$ \\
IL-6 $(\mathrm{pg} / \mathrm{ml})$ & $0.9 \pm 0.2^{\mathrm{b}}$ & $1.1 \pm 0.8$ & $2.9 \pm 3.1^{\mathrm{e}}$ & $2.2 \pm 3.1$ \\
MIP-1 $\alpha(\mathrm{pg} / \mathrm{ml})$ & $268 \pm 150$ & $213 \pm 54$ & $226 \pm 163$ & $348 \pm 145$ \\
MIP-1 $\beta(\mathrm{pg} / \mathrm{ml})$ & $80 \pm 43$ & $66 \pm 23$ & $82 \pm 43$ & $101 \pm 45$ \\
\hline
\end{tabular}

Values are means \pm SD

By one-way ANOVA and post hoc Bonferroni:

${ }_{p}<0.0001$ vs overweight NGT and overweight IGT/type 2 diabetes mellitus (T2DM)

${ }_{p} p<0.05$ vs overweight NGT and overweight IGT/T2DM

${ }^{c} p<0.01$ vs overweight IGT/T2DM

${ }_{p}<0.001$ vs lean NGT

e $p<0.05$ vs lean NGT 
Table 3 Parameters of energy homeostasis in study groups

\begin{tabular}{lcccc}
\hline & Athletes & Lean NGT & Overweight NGT & Overweight IGT/T2DM \\
\hline$n$ & 23 & 13 & 34 & 25 \\
REE (MJ/day) & $8.1 \pm 1.4$ & $7.3 \pm 0.8$ & $7.9 \pm 9.2$ & $8.3 \pm 1.4$ \\
REE/predicted REE (\%) & $111 \pm 17$ & $101 \pm 8$ & $109 \pm 11$ & $111 \pm 12$ \\
RQ & $0.76 \pm 0.05^{\mathrm{a}}$ & $0.80 \pm 0.06$ & $0.83 \pm 0.07$ & $0.84 \pm 0.05$ \\
GOx (mg kg $\left.\mathrm{min}^{-1}\right)$ & $0.81 \pm 0.94^{\mathrm{b}}$ & $1.24 \pm 0.98$ & $1.60 \pm 0.99$ & $1.68 \pm 0.75$ \\
LOx (mg kg $\left.\mathrm{min}^{-1}\right)$ & $1.48 \pm 0.48^{\mathrm{a}, \mathrm{c}}$ & $1.04 \pm 0.45$ & $0.80 \pm 0.42$ & $0.77 \pm 0.35$ \\
\hline
\end{tabular}

Values are means \pm SD

By one-way ANOVA and post hoc Bonferroni:

${ }_{p}<0.001$ vs overweight NGT and overweight IGT/type 2 diabetes mellitus (T2DM)

${ }_{p} p<0.05$ vs overweight NGT and overweight IGT/T2DM

${ }^{c} p<0.01$ vs lean NGT

$G O x$ Glucose oxidation, $L O x$ lipid oxidation

Pro-inflammatory chemokines Fasting serum concentrations of pro-inflammatory chemokines (MCP-1/CCL2, IL6 , MIP-1 $\alpha$, MIP- $1 \beta$; Table 2) were not increased in elite athletes when compared with the other study groups. Statistical analysis revealed that MCP-1/CCL2 was reduced in the athletes in comparison with overweight individuals with IGT and type 2 diabetes mellitus $(p<0.01)$ and that IL- 6 was reduced in the athletes in comparison with all the groups of overweight men (NGT, IGT and type 2 diabetes mellitus; $p<0.001$ ) and in the lean sedentary controls in comparison with the overweight NGT men $(p<0.05)$. MIP- $1 \alpha$ and MIP-1 $\beta$ were not different among groups.

Energy homeostasis REE was not different among groups (Table 3). No difference was found among groups, even when the REE was normalised by the predicted REE based on the classic Harris-Benedict equation (Table 3). In contrast, the oxidative substrate partitioning was found to be different among groups. In detail, the respiratory quotient (RQ) was significantly lower in the athletes when compared with the overweight men (NGT, IGT and type 2 diabetes mellitus; $p<0.001)$ in association with a parallel reduction of glucose oxidation $(p<0.01$; Table 3$)$. Lipid oxidation was higher in the athletes in comparison with the overweight men (NGT, IGT and type 2 diabetes mellitus; $p<0.001)$ and also in comparison with the lean sedentary controls $(p<0.05$; Table 3$)$. No significant difference was detected among the lean sedentary controls in comparison with the overweight men (NGT, IGT and type 2 diabetes mellitus).

Comparison among sprinters, middle-distance and marathon runners When the elite athletes were segregated into sprinters, middle-distance and marathon runners no difference was detected with respect to age, BMI, fasting

Table 4 Laboratory and metabolic features of middle-age, amateur endurance-trained and matched sedentary men

\begin{tabular}{|c|c|c|c|}
\hline & Amateur endurance-trained & Sedentary & $p$ value $^{\mathrm{a}}$ \\
\hline$n$ & 9 & 9 & \\
\hline \multicolumn{4}{|l|}{ Lipid profile } \\
\hline Fasting NEFA (mmol/l) & $0.51 \pm 0.17$ & $0.50 \pm 0.07$ & 0.89 \\
\hline Total cholesterol $(\mathrm{mmol} / \mathrm{l})$ & $5.2 \pm 0.9$ & $4.9 \pm 0.9$ & 0.45 \\
\hline HDL-cholesterol (mmol/l) & $1.7 \pm 0.3$ & $1.3 \pm 0.3$ & 0.05 \\
\hline LDL-cholesterol (mmol/1) & $3.2 \pm 0.9$ & $3.0 \pm 0.8$ & 0.56 \\
\hline Triglycerides $(\mathrm{mmol} / \mathrm{l})$ & $0.7 \pm 0.2$ & $1.2 \pm 1.0$ & 0.22 \\
\hline \multicolumn{4}{|l|}{ Insulin sensitivity } \\
\hline Fasting glucose $(\mathrm{mmol} / \mathrm{l})$ & $4.7 \pm 0.6$ & $4.9 \pm 0.4$ & 0.32 \\
\hline Fasting insulin (pmol/l) & $48 \pm 18$ & $60 \pm 24$ & 0.28 \\
\hline HOMA2-\%B & $113 \pm 40$ & $114 \pm 30$ & 0.92 \\
\hline HOMA2-\%S & $112 \pm 32$ & $89 \pm 31$ & 0.08 \\
\hline HOMA2 & $1.0 \pm 0.4$ & $1.3 \pm 0.5$ & 0.16 \\
\hline Disposition index & $1.3 \pm 0.3$ & $0.9 \pm 0.2$ & 0.04 \\
\hline \multicolumn{4}{|l|}{ Adipokines } \\
\hline Leptin (ng/ml) & $2.7 \pm 1.2$ & $3.5 \pm 1.5$ & 0.25 \\
\hline Adiponectin $(\mu \mathrm{g} / \mathrm{ml})$ & $7.7 \pm 3.0$ & $6.6 \pm 2.9$ & 0.32 \\
\hline Resistin (ng/ml) & $3.6 \pm 0.7$ & $2.9 \pm 0.5$ & 0.03 \\
\hline
\end{tabular}

Values are means \pm SD

andependent-sample $t$-test (two-tailed) 

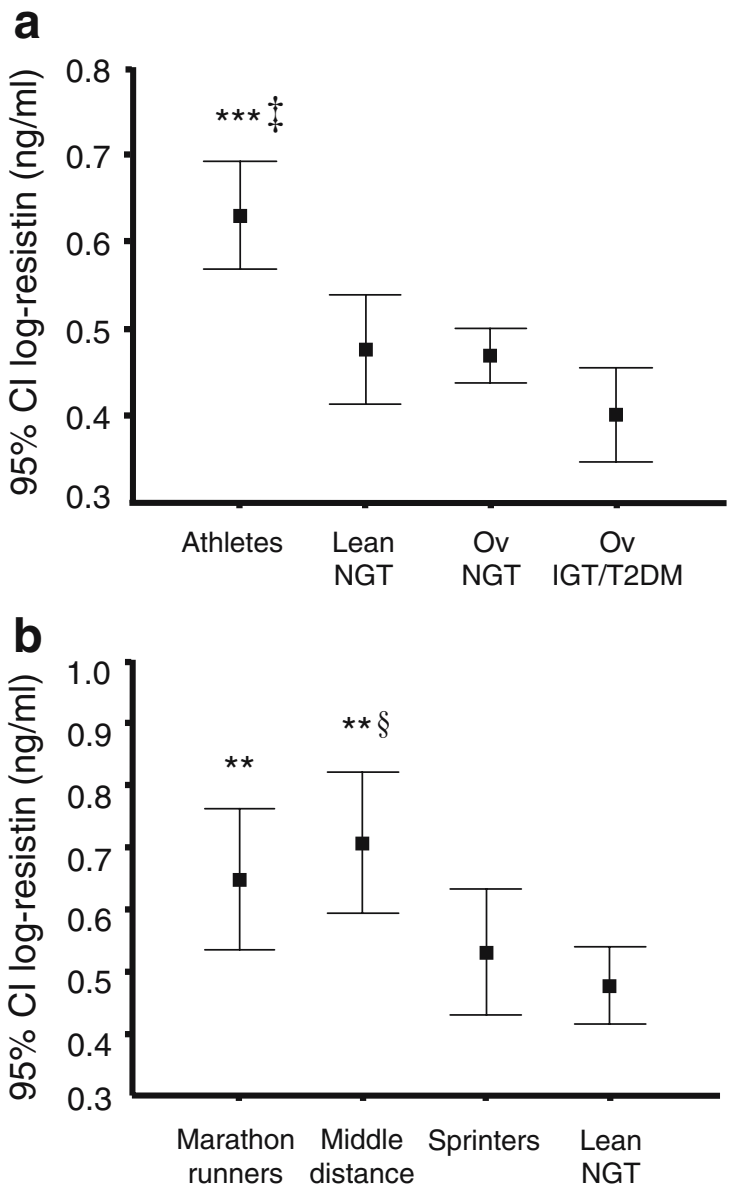

Fig. 1 Serum resistin concentrations in study groups. a Increased log-resistin concentration in athletes in comparison with lean, young, sedentary controls (Lean NGT), overweight individuals with NGT $(O v N G T)$ and overweight individuals with IGT/type 2 diabetes mellitus $(O v I G T / T 2 D M)$. $\$ p<0.0001$ vs overweight NGT and IGT/type 2 diabetes mellitus; $* * * p<0.001$ vs lean NGT; oneway ANOVA and post hoc Bonferroni. b Increased log-resistin concentration in marathon runners and middle-distance runners in comparison with lean, young, sedentary controls (Lean NGT) and the increased log-resistin concentration in middle-distance runners in comparison with sprinters. ${ }^{* *} p<0.01$ vs lean NGT; $\S p<0.05$ vs sprinters; one-way ANOVA and post hoc Bonferroni

plasma glucose, NEFA, lipid profile, insulin sensitivity (WBISI: $9.6 \pm 2.2,9.5 \pm 4.3,10.1 \pm 4.8$ in sprinters, middledistance and marathon runners; $p=0.90$ ) and the parameters of energy metabolism (REE, RQ, glucose and lipid oxidation). No difference was detected also with respect to leptin, adiponectin, IL-6 and the pro-inflammatory chemokines between the subgroups of elite athletes. Despite the lack of difference for all the above-described parameters, only serum resistin was different within the athlete subgroups (ANOVA; $p<0.001$ ). Resistin was higher in the middle-distance and marathon-runner athletes $(5.3 \pm 1.7$ and $4.7 \pm 1.6 \mathrm{ng} / \mathrm{ml})$ in comparison with the lean NGT $(3.1 \pm 0.7 \mathrm{ng} / \mathrm{ml} ; p<0.01)$ as shown in Fig. $1 \mathrm{~b}$, while in the sprinters $(3.5 \pm 0.8 \mathrm{ng} / \mathrm{ml})$ it was not different from that in lean NGT $(p=1.0)$ and was significantly lower in comparison with the middledistance track runners (Fig. 1b; $p<0.05$ ). Plasma homo- cysteine concentrations were not different among groups $(p=0.34)$.

Serum resistin in middle-age amateur endurance-trained men Middle-age, amateur endurance-trained men were characterised by a trend for higher insulin sensitivity (HOMA2-\%S: $p=0.08$; Table 4 ) and had a higher disposition index (Table $4 ; p=0.04$ ) and fasting serum resistin concentration than the sedentary controls (Table $4 ; p=0.03$ ).

General correlation analysis Pearson correlation analysis showed that the serum resistin concentration was associated with age $(r=-0.46 ; p<0.01)$, BMI $(r=-0.29$; $p<0.01)$, glucose oxidation $(r=-0.31 ; p<0.01)$, lipid oxidation $(r=0.38 ; p<0.001)$, RQ $(r=-0.34 ; p<0.01)$, leptin $(r=-0.25 ; p<0.05)$, NEFA $(r=0.20 ; p<0.05)$, glucose $(r=-0.28 ; p<0.05)$, total cholesterol $(r=-0.386 ; p<0.01)$, HDL-cholesterol $(r=0.22 ; p<0.05)$, triglycerides $(r=-0.26$; $p<0.01)$, WBISI $(r=0.43 ; p<0.01)$ and MCP-1/CCL2 $(r=-0.26 ; p<0.01)$. When the partial correlation analysis was performed controlling for the group variable, none of the above-described associations were close to statistical significance, with the exception of fasting plasma NEFA, which remained significantly associated with serum resistin $(r=0.20 ; p<0.03)$, and of plasma glucose, even if it showed only a trend towards a significant association $(r=0.16 ; p=0.062)$. When the relationship between serum resistin and plasma NEFA was analysed separately in normal-weight individuals (Fig. 2a) and overweight individuals (Fig. 2b) a strong association was found in the former $(r=0.27 ; p<0.02)$ but not in the latter $(r=-0.04$; $p=0.74$ ).

\section{Discussion}

This study has produced evidence that resistin is not associated with insulin resistance in humans. Using a novel approach, it tested this possibility in a model of marked insulin sensitivity such as elite athletes. Inspection of the data also revealed that in elite professional athletes serum resistin concentration is increased in comparison with (1) middle-age insulin-resistant men with NGT and/or IGT/type 2 diabetes mellitus and with (2) young, healthy and sedentary men. Moreover, the increased serum resistin concentration was found in athletes undergoing training programmes focused mainly on aerobic endurance exercise and not in those undergoing sustained-power exercise (sprinters).

In spite of the original report linking the adipose tissue hormone resistin with insulin resistance [1] and of subsequent reports showing that in humans this link is controversial $[11-15,27,28]$, our data are in line with a recent report by Utzschneider et al. [29], suggesting that circulating resistin is unlikely to be a major mediator of insulin resistance in men and again supporting the existence of a striking contrast with the observations in rodents [30].

The observational finding that resistin was even higher in elite athletes than in insulin-resistant and healthy controls (Fig. 1a) was unexpected. Therefore, we tested 

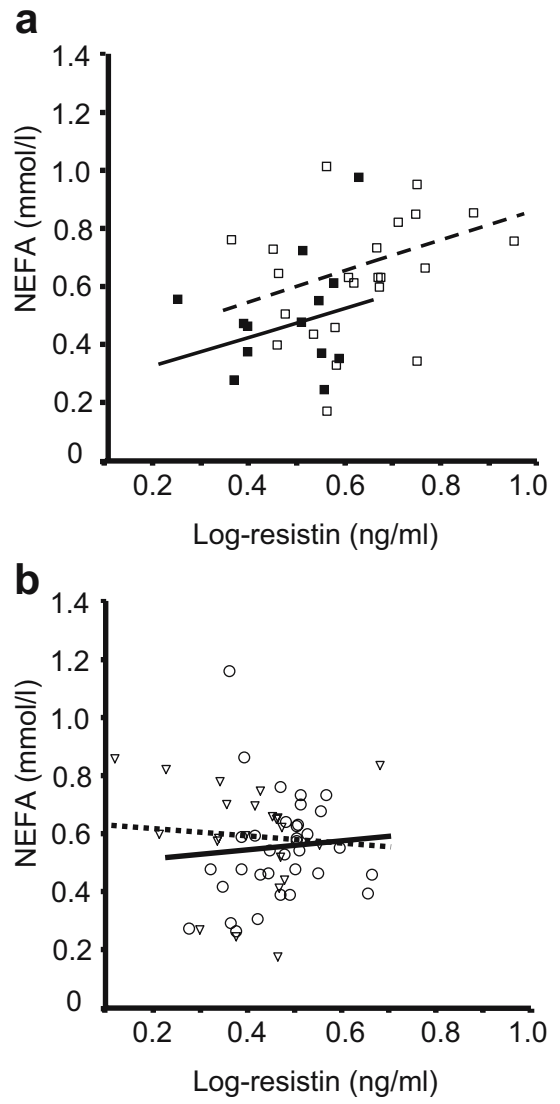

Fig. 2 Association between serum resistin concentration and plasma NEFA. a Scatter-plot correlating the log-resistin and plasma NEFA concentration $(r=0.27 ; p<0.02$ partial correlation controlling for group effect), combining data of the athletes (open squares and broken line) and the lean, young, sedentary volunteers (filled squares and solid line). b Scatter-plot correlating the log-resistin and plasma NEFA concentration ( $r=-0.04 ; p=0.74)$, combining data from the overweight individuals with NGT (open circles and solid line) and overweight individuals with IGT/type 2 diabetes mellitus (open triangles and dotted line)

retrospectively whether this pattern was also reproducible in middle-age, amateur endurance-trained men as an additional, but less extreme model of increased insulin sensitivity in comparison with sedentary individuals and found that the increased serum resistin was confirmed also in non-professional physically fit subjects (Table 4).

We must emphasise that with regard to the interpretation of increased serum resistin in association with exercise training in men, a clear limitation of the present report is the observational approach of our study. We tested whether in the athletes an increased serum resistin concentration reflected ongoing pro-inflammatory events. In fact, resistin's structure is similar to that of proteins involved in inflammatory processes and its serum concentration was found to be associated with coronary artery calcification independently of the C-reactive protein and other inflammatory markers [17]. In addition, exercise activity is known to induce acutely increments of the circulating levels of many pro- and anti-inflammatory cytokines and chemokines [31]. For this reason we measured those markers of 'low-grade inflammation' that have been suggested to be possibly increased in individuals that are chronically exposed to strenuous exercise. We failed to detect a significant association of these markers with the increased serum resistin found in the athletes; in fact, the cyto-chemokine concentrations were not higher than those of the control groups. In detail, MCP-1/CCL2 showed a trend to be lower in athletes than in the overweight IGT/ type 2 diabetic subjects (Table 2), as we have previously reported [32], and although plasma IL-6 increases in an exponential fashion during acute exercise, in the resting state it was lower in the athletes in comparison with the subgroups of overweight individuals (Table 2). The present findings are therefore in agreement with the described long-term anti-inflammatory effect of exercise [26] and apparently argue against the hypothesis that increased resistin in athletes reflects inflammatory events.

A more detailed inspection of our data revealed that the higher resistin levels were found in the athletes whose exercise training must be sustained by the aerobic oxidative system fuelled by macronutrients (aerobic endurance exercise), and were not found in the athletes undergoing a sustained-power exercise training whose performance is based on immediately available intracellular energy depots (ATP and phosphocreatine) (Fig. 1b). We therefore tried to test whether serum resistin concentration was associated with the substrates directly fuelling the aerobic oxidative metabolism and noticed that the plasma NEFA concentration was the only variable which retained a statistical association with the circulating resistin levels when the group effect was taken into account, and this association was more evident when explored in non-obese individuals (Fig. 2a) than in overweight patients with or without IGT/ type 2 diabetes mellitus (Fig. 2b). A comparison with the literature for these aspects is difficult because only recently some effects of resistin on lipid metabolism were reported; a hypertriglyceridaemic effect in vivo in animals $[3,33]$ and a lipolytic effect in vitro [34]. It would appear, therefore, that resistin may be associated with increased NEFA availability in the bloodstream and in this regard it is interesting to note the parallelism with the previously reported paradox of the increased intra-myocellular lipid content despite the high insulin sensitivity reported in athletes [35].

In the elucidation of the mechanisms behind the higher resistin levels in aerobic-endurance athletes many unresolved questions remain, and longitudinal studies showing a direct effect of acute exercise bouts or of chronic exercise training programmes are warranted to fully support the described association and to clarify the impact of aerobic endurance in comparison with sustained-power training programmes. It may be concluded that this study further confirms that increased serum resistin concentration is unlikely to be involved in the pathogenesis of insulin resistance in humans; paradoxically, increased serum resistin was found in extremely insulin-sensitive elite athletes. This work also reported for the first time an independent association between circulating resistin and NEFA concentrations, suggesting that the interactions between resistin and lipid metabolism should be further explored. 
Acknowledgements This study was supported by grants from the Italian Minister of Health (RF98.49, RF99.55, RF01.1831). A. Burska was the recipient of the Marie Curie Host Fellowship of the European Community (contract number HPMT-CT-2001-00329); she is now at the Interfaculty Chair and Department of Laboratory Diagnostics, University of Medicine at Lublin, Lublin, Poland.

\section{References}

1. Steppan CM, Bailey ST, Baht S et al (2001) The hormone resistin links obesity to diabetes. Nature 409:307-312

2. Rajala MW, Obici S, Scherer PE, Rossetti L (2003) Adiposederived resistin and gut-derived resistin-like molecule- $\beta$ selectively impair insulin action on glucose production. J Clin Invest 111:225-230

3. Satoh H, Nguyen MTA, Miles PDG, Imamura T, Usui I, Olefsky JM (2004) Adenovirus-mediated chronic 'hyperresistinemia' leads to in vivo insulin resistance in normal rats. J Clin Invest 114:224-231

4. Muse ED, Obici S, Bhanot S et al (2004) Role of resistin in dietinduced hepatic insulin resistance. J Clin Invest 114:232-239

5. Flier JS (2001) The missing link with obesity. Nature 409:292-293

6. Yang RZ, Huang Q, Xu A et al (2003) Comparative studies of resistin expression and phylogenomics in human and mouse. Biochem Biophys Res Commun 310:927-935

7. Shuldiner AR, Yang R, Gong D-W (2001) Resistin, obesity and insulin resistance - the emerging role of the adipocyte as an endocrine organ. N Engl J Med 345:1345-1346

8. McTernan CL, McTernan PG, Harte AL, Levick PL, Barnett AH, Kumar S (2002) Resistin, central obesity, and type 2 diabetes. Lancet 359:46-47

9. Smith SR, Bai F, Charbonneau C, Janderova L, Argyropoulos G (2003) A promoter genotype and oxidative stress potentially link resistin to human insulin resistance. Diabetes 52:1611-1618

10. Way JM, Gorgun CZ, Tong Q et al (2001) Adipose tissue resistin expression is severely suppressed in obesity and stimulated by peroxisome proliferator-activated receptor gamma agonists. J Biol Chem 276:25651-25653

11. Lee JH, Chan JL, Yiannakouris N et al (2003) Circulating resistin levels are not associated with obesity or insulin resistance in humans and are not regulated by fasting or leptin administration: cross-sectional and interventional studies in normal, insulin-resistant, and diabetic subjects. J Clin Endocrinol Metab 88:4848-4856

12. Degawa-Yamauchi M, Bovenkerk JE, Juliar BE et al (2003) Serum resistin (FIZZ3) protein is increased in obese humans. J Clin Endocrinol Metab 88:5452-5455

13. Heilbronn LK, Rood J, Janderova L et al (2004) Relationship between serum resistin concentrations and insulin resistance in nonobese, obese, and obese diabetic subjects. J Clin Endocrinol Metab 89:1844-1848

14. Jung HS, Youn BS, Cho YM et al (2005) The effects of rosiglitazone and metformin on the plasma concentrations of resistin in patients with type 2 diabetes mellitus. Metabolism 54:314-320

15. Nagaev I, Smith U (2001) Insulin resistance and type 2 diabetes are not related to resistin expression in human fat cells or skeletal muscle. Biochem Biophys Res Commun 285:561-564

16. Patel L, Buckels AC, Kinghorn IJ et al (2003) Resistin is expressed in human macrophages and directly regulated by PPAR gamma activators. Biochem Biophys Res Commun $300: 472-476$
17. Reilly MP, Lehrke M, Wolfe ML, Rohatgi A, Lazar MA, Rader DJ (2005) Resistin is an inflammatory marker of atherosclerosis in humans. Circulation 111:932-939

18. National Diabetes Data Group (1979) Classification and diagnosis of diabetes mellitus and other categories of glucose intolerance. Diabetes 28:1039-1057

19. Zambon A, Hashimoto SI, Brunzell JD (1993) Analysis of techniques to obtain plasma for measurement of levels of free fatty acids. J Lipid Res 34:1021-1028

20. Perseghin G, Scifo P, De Cobelli F et al (1999) Intramyocellular triglyceride content is a determinant of in vivo insulin resistance in humans: a ${ }^{1} \mathrm{H}_{-}{ }^{13} \mathrm{C}$ NMR spectroscopy assessment in offspring of type 2 diabetic parents. Diabetes 48:1600-1606

21. Fermo I, Arcelloni C, Mazzola G, D’Angelo A, Paroni R (1998) High-performance liquid chromatographic method for measuring total plasma homocysteine levels. J Chromatogr B Biomed Sci Appl 719:31-36

22. Matsuda M, De Fronzo RA (1999) Insulin sensitivity indices obtained from oral glucose tolerance testing. Comparison with the euglycemic insulin clamp. Diabetes Care 22:1462-1470

23. Wallace TM, Levy JC, Matthews DR (2004) Use and abuse of HOMA modeling. Diabetes Care 27:1487-1495

24. Weir JB (1949) New methods for calculating metabolic rate with special reference to protein metabolism. J Physiol (Lond) 109:1-9

25. Johnson MM, Chin R, Haponik EF (1999) Nutrition, respiratory function, and disease. In: Shils ME, Olson JA, Shike M, Ross AC (eds) Modern nutrition in health and disease. Williams \& Wilkins, 1473-1490

26. Frayn KN (1983) Calculation of substrate oxidation rates in vivo from gaseous exchange. J Appl Physiol 55:628-634

27. Youn BS, Yu KY, Park HJ et al (2004) Plasma resistin concentrations measured by enzyme-linked immunosorbent assay using a newly developed monoclonal antibody are elevated in individuals with type 2 diabetes mellitus. J Clin Endocrinol Metab 89:150-156

28. Pagano C, Marin O, Calcagno A et al (2005) Increased serum resistin in adults with Prader-Willi syndrome is related to obesity and not to insulin resistance. J Clin Endocrinol Metab 90:4335-4340

29. Utzschneider KM, Carr DB, Tong J et al (2005) Resistin is not associated with insulin sensitivity or the metabolic syndrome in humans. Diabetologia 48:2330-2333

30. Arner P (2005) Resistin: yet another adipokine tells us that men are not mice. Diabetologia 48:2203-2205

31. Petersen AMK, Pedersen BK (2005) The anti-inflammatory effect of exercise. J Appl Physiol 98:1154-1162

32. Piemonti L, Calori G, Mercalli A et al (2003) Fasting plasma leptin, tumor necrosis factor-a receptor 2 and monocyte chemoattracting protein 1 concentration in a population of glucose-tolerant and glucose-intolerant women. Diabetes Care 26:2883-2889

33. Sato N, Kobayashi K, Inoguchi T et al (2005) Adenovirusmediated high expression of resistin causes dyslipidemia in mice. Endocrinology 146:273-279

34. Ort T, Arjona AA, MacDougall JR et al (2005) Recombinant human FIZZ3/resistin stimulates lipolysis in cultured human adipocytes, mouse adipose explants, and normal mice. Endocrinology 146:2200-2209

35. Goodpaster BH, He J, Watkins S, Kelley DE (2001) Skeletal muscle lipid content and insulin resistance: evidence for a paradox in endurance-trained athletes. J Clin Endocrinol Metab $86: 5755-5761$ 